of Caesarean sections. You learn that Marc Chagall was thought to be dead at birth, but revived when he was dropped into a bucket of ice water. The chapter and book end with a lively presentation of aspects of being a new mother, and the final word is directed at the future of human pregnancy in the context of contemporary reproductive technology.

Bainbridge has written a highly informative, enlightening and amusing book on human pregnancy. It certainly will be appreciated by a wide audience.

\section{Malaria: Poverty, Race, and Public Health in the United States}

\author{
by Margaret Humphreys \\ Johns Hopkins University Press \\ \$41.50, 256 pp, 2001
}

ReViewed by Allan Saul

Malaria Vaccine D evelopment Unit, National Institutes of Health, Rockville, Maryland, USA

I eagerly started Malaria: Poverty, Race, and Public Health in the United States, looking forward to the insights into malaria control that could be gained from the successful experience of the United States. I wanted to see what lessons could be applied to the development of malaria-control programs in similarly rural, poor and relatively low endemic areas and how these compared to my own experiences in developing sustainable malaria control programs in The Philippines. However, my expectations were quickly lowered by reading, in the Introduction, Margaret Humphreys' rationale for this book: "I had once hoped that this research project might result in more than an historical narrative, that it might actually bear relevance for the bel eaguered international malaria community". Instead she conveys her disappointment that, “The history of malaria's disappearance from the United States has few lessons to offer for those struggling to combat the disease in its contemporary tropical homes."

After having read the book, I disagree. The difference of opinion may relate to the author's limited knowledge of the biology of the parasite and its host, the nuances of the global epidemiology of malaria and modern approaches to control programs. The book relates a dismal tale of how malaria control efforts have failed globally and how, "No new control strategy has emerged in recent years to replace the faulty DDT spraying program...Malariologists in developing countries are turning increasingly to low-technology solutions such as insecticide-treated bed nets, as nothing else is available."

True, malaria continues to kill huge numbers of children, to sicken a high proportion of the world's population and to impose major direct and indirect economic burdens on the developing world, but there have been successes since DDT. The near elimination of malaria in China from a peak of tens of millions of cases annually in the 1970s is one such success. Other steady, albeit less spectacular, gains in specific programs in Southeast Asia, South Asia, Central and South America also come to mind. Increasingly, new control strategies in endemic areas, such as those promoted under the umbrella of the WHO Roll Back Malaria Program, rely on integrated programs to empower communities to provide access to better health care, better health education, early diagnosis and treatment, wealth-generation schemes so people can afford health care and lifestyle changes to reduce risk, as well as anti-mosquito measures such as bed nets and spraying. These are lowtechnology perhaps, but increasingly sophisticated in their development of multifactor and sustainable programs.

Apart from the pessimistic big picture of this story, there are also numerous, usually trivial, but annoying errors. For example, in describing the lifecycle of malaria parasites, there are at least six significant errors in one paragraph. These do not inspire confidence in the scholarship behind this book.

If one accepts that Malaria is simply "an historical narrative" and ignores the minutiae, then it offers a fascinating story of the spread of malaria through the USA following its introduction in the 17th century, through its greatest geographical coverage in the 19th century. The story culminates in the steady retreat and eventual elimination of malaria as an endemic disease in the 1950s. The first 250 years of malaria in the USA are covered in Chapters 1 and 2 , whereas most of the remainder of the book details the social and medical impact of malaria in the southern states during the first half of the 20th century.

In Chapter 4, there is an interesting analysis of the effective 'malaria tax' imposed on industry and how the realization of this economic impact led to the mobilization of re sources to control the disease, a topic of considerable importance in current malaria-control programs. A highlight of the book is the anecdotal but relevant insight into knowledge, attitudes and practices toward provision of health services in general and malaria in particular, through the unique documentation generated in the 1930s by the Federal Writers' Project. These accounts chronicle a system in transition from old beliefs of malaria as an environmental hazard (contracted by 'bad water'), to more modern ideas of malaria as a contagion carried from person to person by mosquitoes; the impact of the education system in the introduction of new ideas; and the constraints imposed by poverty on the ability to act on the information even when understood.

These are topics with an uncanny relevance to contemporary control programs tailored to low-to-moderate endemic areas. These efforts face similar obstacles with regard to economics, health-seeking practices and beliefs, as well as the more technical issues of vector control and treatment of patients. Even if the only lesson to be learnt from the US is that malaria can be beaten, then this alone is important.

Humphreys poses the question, "Who killed malaria [in the USA]?" and proposes a novel, but plausible, explanation for the coup de grace, which I won't spoil by disclosing here. However, even in unraveling this mystery she warns, "that you may be wiser at the end, but still not sure." My advice for readers of this book: Don't let the limits imposed by the author spoil a good story of considerable relevance for contemporary malariacontrol programs. 